\title{
Evaluación del Impacto Normativo (EIN) y Evaluación de Políticas Públicas
}

\author{
Carlos Román del Río \\ Ana Cirera León
}

\section{EL ENFOQUE INTEGRAL DE LA EVALUACIÓN DE POLÍTICAS PÚBLICAS}

La evaluación de políticas públicas consiste en la aplicación sistemática de los métodos de investigación y de las técnicas cuantitativas y cualitativas, propios de las ciencias sociales, a la valoración de las intervenciones del Estado en la vida económica y social. El cuadro siguiente gráfico se presenta el enfoque de evaluación que se desarrolla en el artículo.

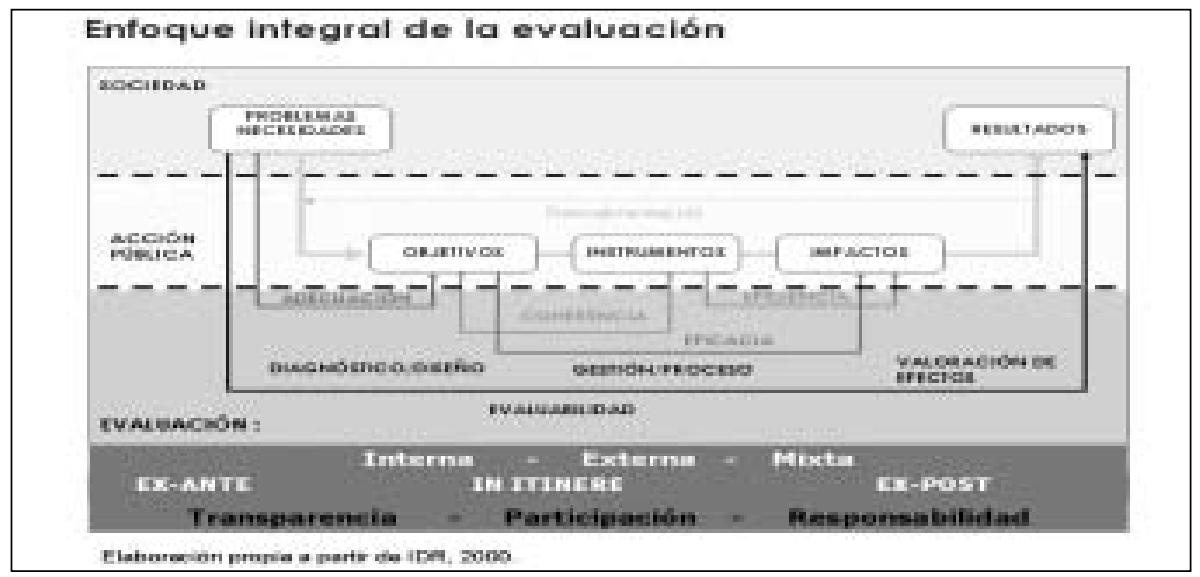

Fuente: Adaptado de la Guía para la Evaluación de las Políticas Públicas, IDR, 2000.

La Administración Pública, en sus diferentes niveles de descentralización funcional y territorial, desarrolla políticas y presta servicios cumpliendo mandatos constitucionales y a partir de normas previamente aprobadas, reglamentarias o legales, de acuerdo con el principio de legalidad contemplado en el art. 9.3 de la Constitución Española. 
Por tanto, las normas son, como también señala el informe del Grupo Mandelkern sobre Mejora de la Regulación ${ }^{1}$, "esenciales para alcanzar los objetivos de las políticas públicas". De acuerdo con ello, la mejora de la regulación no trata de eliminar las normas de una manera irreflexiva, sino de garantizar que se opte por elaborar normas cuando resulte adecuado y, además, que cuando se regule, las normas sean de calidad.

Por ello, la mejora de las normas jurídicas puede ser entendida como la política centrada en la búsqueda de la mejora y simplificación del entorno normativo, y no como una política destinada a desregular. La mejora de la normativa busca acrecentar la calidad de la normativa que se va a dictar en el futuro, y de la ya existente, lo que puede o no dar lugar a la eliminación de normas jurídicas.

En todo caso, la actuación pública parte, o debería partir, de un análisis riguroso de la realidad sobre la que ha decidido actuar. Esto supone identificar y caracterizar problemas y necesidades, así como sus causas y consecuencias, agentes implicados e impactos esperados. Sólo a partir de la correcta identificación de estas variables es posible proponer objetivos, medidas y normas que contribuyan a la superación de la problemática que se pretende resolver.

En un modelo de planificación estratégica, la imagen formada de la realidad que se quiere modificar se contrapone con la imagen ideal a la que se quiere llegar. Siempre que se desee que el proceso de ajuste necesario definido por la política o norma no sea traumático y no provoque conflictos sociales de consecuencias difícilmente reparables, es recomendable, además de una planificación integrada que atienda a la solución jerarquizada de los estrangulamientos, un permanente diálogo político y social. Por muy obvio que pueda parecer un determinado problema, es importante sistematizar el conocimiento y la visión de las instituciones, públicas y privadas, implicadas en la gestión y ejecución de las actuaciones, así como de otras organizaciones que representan a colectivos de ciudadanos.

Participación, consenso y compromiso en la definición de problemas, y establecimiento de normas, objetivos y estrategias son los ejes fundamentales para garantizar el éxito de la planificación y de la intervención pública, así co-

\footnotetext{
${ }^{1}$ Informe Final del Grupo Mandelkern sobre Mejoras de la Regulación. Noviembre de 2001. Consultable en http://www.igsap.map.es/cia/orgpub/mandelkern1.htm
} 
mo para diseñar los sistemas de información y realizar los procesos de evaluación que toda acción pública debe desarrollar a los largo de su ciclo de vida.

En este sentido, se puede establecer que la diferencia principal entre la evaluación del impacto normativo (EIN) y la evaluación de políticas públicas es, precisamente, el objeto de análisis o estudio.

Las EIN centran su atención exclusivamente en las normas jurídicas (en sus diferentes categorías o rangos) y, en este sentido, se pueden considerar una herramienta de apoyo a la toma de decisión normativa; sobre todo, si se realiza a través del denominado modelo empirico de decisión política ${ }^{2}$, que basa los criterios decisorios en los hechos contrastados o efectos esperados de la norma y en el análisis de los parámetros de acción.

Así, se puede definir la evaluación del impacto normativo como una técnica que trata, mediante la evaluación de las normas, de estimar los impactos que éstas tendrán en el contexto socioeconómico para conocer los aspectos positivos y negativos de su aprobación o de su aplicación. Los criterios sobre los que se basa la evaluación son los costes, los beneficios y los riegos de la aprobación de la norma ${ }^{3}$.

Por su parte, la evaluación de políticas públicas, sobre todo cuando se realiza desde una perspectiva ex-ante, tiene como finalidad valorar las consecuencias e impactos que sobre la realidad socioeconómica puede tener la aplicación de una determinada política o acción pública. Es decir, se trata de establecer, con tanta certeza como sea posible, si la intervención producirá o no los efectos que busca, cuál será la repercusión del programa y cuál será la eficacia alcanzada en relación con las metas marcadas.

De esta forma, la evaluación se convierte en una herramienta al servicio del gestor y del político para mejorar sus intervenciones y para dar coheren-

\footnotetext{
${ }^{2}$ En general, los métodos usados por los países de la OCDE para tomar sus decisiones normativas se pueden clasificar en los siguientes: los que buscan la opinión de expertos (internos o externos), los consensuados con distintos grupos de interés, los políticos, los comparativos (benchmarking) y los empíricos. "An Overview of Regulatory Impact Analysis in OECD Countries". Scott H. Jacobs. Consultable en http://www.oecd.org/dataoecd/21/59/35258828.pdf.

3 "Better Policy Making: A Guide to regulatory Impact Assessment". Regulatory Impact Unit. London, 2003. Consultable en: http:www.cabinet-office.gov.uk/regulation.
} 
cia al proceso de transformación del "discurso político" en un plan de actuación. Asimismo, pretende ofrecer un marco de análisis en el que validar las etapas del proceso de planificación, esto es, desde que se decide intervenir hasta que se manifiestan los impactos, valorando el diseño, gestión, aplicación y resultados de las intervenciones programadas.

Por ello, su objeto de análisis son, sobre todo, los planes específicos que se aprueban desde el ejecutivo para implementar las decisiones políticas. Esta delimitación del alcance no es excluyente, pues en numerosas ocasiones los planes se desarrollan a través de reglamentos, que son documentos normativos.

Quienes toman las decisiones políticas no siempre tienen que buscar la racionalidad tecnoeconómica; en muchas ocasiones, se persiguen soluciones socialmente aceptables o políticamente convenientes. Por ello, la evaluación también ha de plantearse como objeto aspectos tales como los siguientes:

- Gestión eficaz de los recursos.

- Difusión de la cultura de la responsabilidad entre los gestores de programas.

- Consideración del receptor de la política como usuario.

- Preocupación por conocer el impacto de la política.

Otro ámbito diferente es el análisis de la calidad de las normas. Las normas jurídicas, por mandato constitucional, deben ser racionales, eficaces, eficientes, y deben proteger los derechos constitucionales y permitir también su adecuado desarrollo. Tal y como analiza el Profesor Julio Ponce ${ }^{4}$, el estado actual de la ciencia jurídica permite responder a estas cuestiones.

En primer lugar, hay que determinar qué es la calidad normativa. Para poder establecer la calidad, o la falta de calidad de una norma jurídica, se ha de contar con estándares o criterios de calidad que permitan la comparación. En la actualidad, estos estándares son de dos tipos: formales, referidos a la estructura e inteligibilidad de la norma, estudiados y desarrollados por la deno-

\footnotetext{
${ }^{4}$ Documento de trabajo para Sesiones Formativas del IDR. Febrero 2005.
} 
minada teoría de la legislación; y materiales, referidos a los impactos que la norma jurídica tiene en el mundo real, en la esfera personal y patrimonial de los ciudadanos y de las empresas.

Desde la primera perspectiva, existen desarrollos científicos y documentos jurídicos, como las Directrices sobre la forma y estructura de los anteproyectos de Ley, aprobadas por acuerdo del Consejo de Ministros de 18 de octubre de 1991 (BOE № 276, de 18 de noviembre de 1991), y ahora sustituidas por las nuevas Directrices de Técnica Normativa aprobadas por el Consejo de Ministros de 22 de julio de 2005, (BOE № 180, de 29 de julio de 2005). Así mismo, encontramos algunos ejemplos autonómicos, como la Guía para la Elaboración y Control de Disposiciones de Carácter General, aprobada por Acuerdo del Consejo de Gobierno del Principado de Asturias el 2 de junio de 1992, o las Directrices para la Elaboración de Proyectos de Ley, Decretos, Órdenes y Resoluciones, aprobadas por Acuerdo del Consejo de Gobierno de la Comunidad Autónoma del País Vasco, el 23 de marzo de 1993.

Este tipo de análisis trata de verificar la calidad de la norma en el sentido de que esté correctamente redactada, que no sea arbitraria, que pueda ser efectivamente respetada por los destinatarios y no vulnere (y sí permita) el desarrollo de los derechos constitucionales.

Desde la perspectiva material, se trata de realizar una evaluación del impacto normativo, ya que el incremento de la calidad de las normas es un bien público en sí mismo en la medida en que "refuerza la credibilidad del gobierno y contribuye al bienestar de los ciudadanos, las empresas y demás intervinientes" (Informe Mandelkern). Para conocer la calidad de las normas y, en su caso, mejorarla, es requisito imprescindible evaluarlas, es decir, medir los efectos, los impactos de la normativa en el mundo real.

Unas normas de calidad previenen la imposición de barreras innecesarias a los empresarios, ciudadanos y Administraciones Públicas ahorrando, por tanto, tiempo y dinero. Por otro lado, la aplicación de este tipo de normas resulta menos problemática para las Administraciones Públicas y su cumplimiento más fácil para los ciudadanos. Por estas razones, resulta clave para el interés público incrementar la calidad de las normas.

En todo caso, ambos tipos de evaluación (de la norma y la de políticas públicas) no sólo no son incompatibles, sino que pueden y deben ser concurrentes. Antes de dar paso a la publicación de una norma (es decir, al impulsar la normativa, al redactarla y al ser debatida, en su caso, en el Parlamento) 
o poner un marcha un plan de actuación pública, puede evaluarse qué impactos tendrán y, en función de tal evaluación, decidir, incluso, que puede ser más conveniente no promulgar la norma o ejecutar el plan, por ejemplo.

Por ello, uno de los objetivos de este tipo de evaluación es facilitar la integración y coordinación de los objetivos políticos. Los programas que se diseñan y ejecutan encajan en la agenda política como una pieza de un puzzle que persigue, o al menos así debe serlo a priori, objetivos comunes y más amplios que están intrínsecamente relacionados. (Osuna, Vélez, Cirga y Murciano, 2006).

La naturaleza de los impactos provocados por las políticas se inserta en una compleja realidad socioeconómica, que puede no ser correctamente apreciada si no se dispone de un marco conceptual preciso que ayude a situar el instrumento frente a la problemática socioeconómica a la que responde.

Por este motivo, no deben tratarse las políticas de manera aislada, porque están presentes y afectadas por varios ámbitos de actuación pública y, en ese sentido, deben contribuir a otros fines como el crecimiento económico y el empleo, a la política de cohesión social y protección de los colectivos más desfavorecidos, a políticas redistributivas, etc. De este modo, un aspecto clave es evaluar para asegurar la congruencia entre los ámbitos que cada política o norma regula, evitando conflictos que disminuyan la eficacia y eficiencia de la intervención, o que incluso puedan llegar a anularla.

Además, las normas, políticas y programas públicos cada vez son más complejos ya que son de naturaleza intergubernamental e involucran a actores diversos y heterogéneos. Su puesta en marcha implica la participación de una multiplicidad de instituciones, gestores y beneficiarios. Asimismo, la articulación de las distintas instituciones públicas que confluyen en la ejecución y gestión de las políticas públicas se configura como un sistema multinivel de gobierno que añade complejidad al proceso de elaboración, ejecución, seguimiento y evaluación de las actuaciones públicas.

La descentralización, que a priori parece beneficiosa puesto que se busca la adaptación de la política a cada territorio, requiere coordinación y coherencia en las actuaciones públicas para evitar duplicidad y efectos indeseados. El proceso descentralizador induce a la reflexión sobre la efectividad real del funcionamiento del sistema administrativo. En esencia, el traspaso de las competencias de las políticas se justifica por la mayor eficacia y eficiencia de su gestión desde una Administración más próxima y conocedora de la realidad territorial de su ámbito de actuación. 
El conocimiento de la realidad que funcionarios, empleados públicos, y agentes sociales y económicos poseen, puede garantizar, en principio, que las estrategias públicas se desarrollen con éxito. El estudio de los mecanismos de coordinación y comunicación, y la corresponsabilidad de las distintas Administraciones competentes y agentes involucrados son clave a la hora de determinar el funcionamiento de las actuaciones acometidas por cada nivel de gobierno y organización responsable, de acuerdo con sus competencias.

De esta manera, y como afirma Prats ${ }^{5}$, "la eficacia y la legitimidad política se basa en la calidad de la interacción entre los distintos niveles de gobierno y entre éstos y las organizaciones empresariales y de la sociedad civil". Por ello, el diseño de la evaluación debe incorporar la multiplicidad y heterogeneidad de actores, en un intento de facilitar el establecimiento de criterios y objetivos comunes haciendo partícipes a los implicados en el gobierno de su propio desarrollo.

La evaluación de políticas públicas y la evaluación del impacto normativo, además, se justifica por los siguientes motivos:

- Legitimar la intervención. El sector público debe estar al servicio de los ciudadanos y rendir cuentas ante ellos de sus actuaciones. Esta rendición de cuentas debe realizarse, por un lado, a través de mecanismos de control de la gestión de los recursos públicos; y por otro, mediante la evaluación de la adecuación y eficacia de las políticas públicas en la corrección de las situaciones que provocaron la necesidad de intervención.

- Aumentar la transparencia del sector público. La evaluación no sólo proporciona información a todos aquellos interesados en conocer cómo se elaboran y ejecutan las políticas y normas y cuáles son sus resultados, sino que proporciona el conocimiento veraz y riguroso con que una Administración moderna debe tomar decisiones.

- Hacer efectiva la participación de los ciudadanos en la toma de decisiones. La evaluación proporciona cauces y procedimientos para la participación de los ciudadanos en las fases de planificación, segui-

\footnotetext{
${ }^{5}$ Joan Prats (2004) "Las transformaciones de las administraciones publicas de nuestro tiempo", en Instituciones y Desarrollo [http://www.iigov.org; 16/07/2004].
} 
miento y evaluación de los resultados de las políticas. La evaluación se convierte, así, en un instrumento al servicio de la democracia.

- Mejorar los programas de intervención pública. Las políticas deben ser evaluadas en función de su capacidad para contribuir, por sí solas o mediante las sinergias que pueden producir con otras medidas, a alcanzar los objetivos de la intervención en la que se inserten. La evaluación mejora la eficacia porque proporciona un mecanismo de retroalimentación en la planificación, mejora el conocimiento sobre acciones futuras, y permite detectar y corregir los errores y efectos no deseados.

- Introducir mayor racionalidad y coherencia en la toma de decisiones. La evaluación pone a disposición de los responsables políticos y los ciudadanos información objetiva e imparcial sobre la coherencia, el funcionamiento y los resultados de las políticas. La evaluación debe incorporarse desde el primer momento de la planificación, examinando, de modo sistemático, la idoneidad de la lógica y de las relaciones de causalidad, los impactos previstos, y la adecuación de los sistemas articulados para la ejecución de la política.

- Impulsar el aprendizaje y la innovación en la actuación del sector público. La evaluación exige analizar el grado de aceptación y la capacidad de los responsables políticos, gestores y técnicos para la puesta en funcionamiento de nuevas medidas, su seguimiento y control para responder a problemas emergentes. Proporciona instrumentos para conocer el significado y repercusiones de sus actuaciones, y constituye, de esta forma, un acicate para el cambio y la mejora constantes de la gestión pública.

Estos motivos exigen el tránsito de una evaluación ajena al proceso político y al margen de una tabla axiológica referencial de la organización social, a una evaluación inmersa en el proceso político e involucrada con sus valores.

La evaluación debe constituirse como una herramienta estratégica para una nueva gobernanza y una buena Administración (en los términos del Art. II-101 del Tratado por el que se establece una Constitución para Europa), y como signo de innovación y modernidad en la gestión pública.

Además, y en estos momentos, un aspecto clave es que el interés por estas cuestiones no debe ser sólo teórico, porque "uno de los grandes retos de las 
ciencias sociales en el momento actual consiste en redefinir la intervención pública en las sociedades maduras, participativas y democráticas, lo que hace que la legitimación de la intervención pública pase ineludiblemente por garantizar la transparencia en la gestión y ejecución de sus actuaciones, y no exclusivamente en el cumplimiento de la legalidad y la regulación social a través de las normas" 6 .

Por último, pero no por ello menos importante para que la evaluación sea un proceso operativo debe estar integrada en la gestión diaria de los centros directivos de la Administración. No debemos olvidar que su objetivo último es servir a los gestores del gasto como instrumento para la mejora de la gestión de servicios públicos, de modo que debe garantizarse que los resultados de las evaluaciones sean útiles y utilizables.

El enfoque metodológico que consideramos más apropiado para abordar estos procesos de evaluación se caracteriza, en términos generales, por la aplicación de lo siguiente:

- Un enfoque pluralista y participativo. Como se ha venido explicitando, la evaluación debe entenderse como un proceso de diálogo y negociación que promueva la participación activa de los agentes implicados.

- La adaptación del enfoque del análisis de redes para determinar las relaciones entre los actores, la estructura de la red que configuran y su funcionamiento ${ }^{7}$ (Vélez, 2006). Para su aplicación se debe estudiar la posición central de los actores, los círculos sociales, las subredes; en definitiva, las relaciones que mantienen los actores de la cooperación.

La pertinencia de este enfoque reticular se fundamenta en el supuesto de que tal estructura es un condicionante principal de la formación de interés colectivo, de la definición de objetivos, de la constitución de las organizaciones y alianzas, y de la planificación y viabilidad.

\footnotetext{
${ }^{6}$ Joan Subirats (1996). "Análisis de políticas públicas y gestión pública: promesas y riesgos de una vida en común”, en Quim Brugué. y Joan Subirats (1996). Lecturas de Gestión Pública. Ministerio de Administraciones Públicas. Madrid.

${ }^{7}$ Carmen Vélez (2006). "Enfoques para la colección de la Política de Cooperación: una primera reflexión”. XI Congreso Internacional del Clad. Guatemala, noviembre, 2006.
} 
- Una orientación analítica, formativa y conclusiva. La evaluación debe vincular los aspectos críticos del programa o norma con la gestión, la realización y los resultados para, revelando las relaciones causales e interrelaciones que existen entre ellos, calificar la eficacia y eficiencia de sus acciones y programas.

- Una estrategia basada en la utilización de los resultados del proceso de evaluación. Por tanto, debe producir información útil a todos los que participan en el programa y, fundamentalmente, a sus destinatarios, permitiendo el aprendizaje y difusión de buenas prácticas.

En conclusión, apostar por realizar evaluaciones, de la norma o de las políticas, requiere asumir un compromiso político cuyos beneficios se deben traducir en una gestión innovadora, eficaz y eficiente de los recursos, la difusión de una cultura de responsabilidad entre los gestores de los programas, la consideración del receptor de la política como usuario, el conocimiento del impacto de la política, y la necesidad de incorporar las percepciones de los beneficiarios y participantes.

De esta forma, se entiende la evaluación como parte de un proceso colectivo, tendente a solucionar problemas, que requiere que los implicados en los programas públicos sean los que juzguen la acción pública.

Como hemos dicho, las actuaciones que se ejecutan desde diversas instituciones producen efectos interrelacionados y complejos que precisan de la realización de evaluaciones que, sin incurrir en costes excesivos, sean flexibles y participativas, capaces de detectar y explotar los beneficios de la retroalimentación, y que permitan conocer el grado de satisfacción de los beneficiarios y los ciudadanos con las políticas. 


\section{BIBLIOGRAFÍA BÁSICA}

- "Better Policy Making: A Guide to regulatory Impact Assessment". Regulatory Impact Unit. London, 2003. Consultable en:

http:www.cabinet-office.gov.uk/regulation

- Cerrillo, A. (Coord.) (2005). La Gobernanza hoy: 10 textos de referencia. Instituto Nacional de Administración Pública. Madrid.

- Informe Final del Grupo Mandelkern sobre Mejoras de la Regulación. Noviembre de 2001. Consultable en http://www.igsap.map.es/cia/orgpub/mandelkern1.htm.

- Osuna, J.L. y Márquez, C. (Coord.). (2000). Guía para la evaluación de las políticas públicas. IDR. Sevilla.

- Osuna, J.L., Vélez, C., Cirga, A., Murciano, J. (2006). "El enfoque de evaluación: un triángulo complejo”. En Revista Económica nº 60. Universidad del País Vasco.

- Ponce, Julio. "Evaluación de la Calidad Formativa". Documento de trabajo para Sesiones Formativas del IDR. Febrero 2005.

- Ponce, J. (2001). Deber de buena administración y derecho al procedimiento administrativo debido. Las bases constitucionales del procedimiento administrativo y del ejercicio de la discrecionalidad. Lex Nova, Valladolid.

- Prats, Joan (2004). "Las transformaciones de las administraciones publicas de nuestro tiempo" en Instituciones y Desarrollo [http://www.iigov.org; 16/07/2004].

- Prats, Joan (2005). De la Burocracia al Management, del Managemet a la Gobernanza. Instituto Nacional de Administración Pública. Madrid.

- Scott H. Jacobs. "An Overview of Regulatory Impact Analysis in OECD Countries". Consultable en http://www.oecd.org/dataoecd/21/59/35258828.pdf

- Subirats, J. (1996). "Análisis de políticas públicas y gestión pública: promesas y riesgos de una vida en común”, en Brugué, Q. y Subirats, 
J. (1996). Lecturas de Gestión Pública. Ministerio de Administraciones Públicas. Madrid.

- Subirats, J. (1994). Análisis de políticas públicas y eficacia de la Administración. Ministerio de Administraciones Públicas. Madrid.

- Vélez, C. (2006). Enfoques para la evaluación de la Política de Cooperación: una primera aproximación". XI Congreso Internacional del Clad. Guatemala, noviembre, 2006. 